\title{
Tópicos do Método da Razão de Bowen
}

\author{
Demonstration of the Bowen Ratio Method
}

\begin{abstract}
Osvaldo Alves Pereira*a; Victor Hugo de Moraes Danelichen ; Noel Flávio Costa Ferreira ${ }^{\text {b; }}$ Eduardo Nogueira dos Santos $^{\text {c }}$, Jonathan Willian Zangeski Novais ${ }^{\mathrm{a}}$; Henry Helber Calazans Camargo ${ }^{\text {a }}$, Benedito Felipe da Silva Filho ${ }^{\mathrm{a}}$
\end{abstract}

\author{
aUniversidade de Cuiabá, Programa de Pós-Graduação Stricto Sensu em Ciências Ambientais. MT, Brasil. \\ 'Instituto Federal de Mato Grosso. MT, Brasil. \\ 'Universidade de Cuiabá. MT, Brasil. \\ *E-mail: osvaldo.pereira@kroton.com.br
}

\begin{abstract}
Resumo
Este trabalho teve como objetivo mostrar a dedução física e matemática do método de Bowen na conversão de fluxo de calor latente em unidade de volume de água. A evapotranspiração é o processo natural de fluxo de água para atmosfera. Dentre as técnicas meteorológicas existentes, o método de Bowen consiste na razão entre os fluxos de calor latente (energia destinada para mudança de estado físico da água) e sensível (energia destinada para mudança de temperatura) emitidos por uma superfície durante o processo de evaporação e transpiração das plantas, em função dos gradientes da pressão de vapor e da temperatura observados sobre a superfície. Além disso, o método está fundamentado no princípio de conservação de energia, com fundamentos matemáticos relativamente simples e com modesto aparato instrumental.
\end{abstract}

Palavras-chave: Fluxo de Calor. Micrometeorologia. Perda de Água. Atmosfera.

\begin{abstract}
This work aimed to show the physical and mathematical deduction of the Bowen method in the conversion of latent heat flow into a unit of water volume. Evapotranspiration is the natural process of flowing water into the atmosphere. Among the existing meteorological techniques, the Bowen method consists of the ratio between the latent heat flows (energy destined to change the physical state of the water) and sensitive (energy destined to change the temperature) emitted by a surface during the evaporation process and plant transpiration, depending on the vapor pressure and temperature gradients observed on the surface. In addition, the method is based on the principle of energy conservation, with relatively simple mathematical foundations and with modest instrumental apparatus.
\end{abstract}

Keywords: Heat Flow. Micrometeorology. Loss of Water. Atmosphere.

\section{Introdução}

Segundo Mu et al. (2011), o processo mais importante de transferência de água da superfície para atmosfera é por meio da evapotranspiração. O termo evapotranspiração (ET) foi criado por Thornthwaite (1946), e significa a transferência em conjunto da evaporação d'água de uma superfície mais a transpiração das plantas. Conhecer os dados de (ET) de uma determinada cultura se torna necessário para caracterização fisiológica de plantas, manejo e dimensionamento de projetos de irrigação. Que em geral são obtidos utilizando-se modelos que estimam a ET a partir de dados meteorológicos.

A determinação da (ET) de superfícies vegetais por métodos micrometerológicos apresentam vantagens em relação aos métodos de medida direta, pois, associado a determinação de ET, podem prover informações dos fluxos de calor latente (vapor de água) e de outras gases (Batista $e t$ al., 2015).

Monteith (1981), introduziu as funções de resistências aerodinâmicas e resistências do dossel vegetal, eliminando a função vento da equação original de Penman, hoje chamada de Penman-Monteith, vem sendo utilizada pela Food end
Agricuture Organization (FAO) como equação padrão para a estimativa de evapotranspiração real Allen et al. ( 1998).

Os estudos de transferências dos fluxos de entidades como o calor latente, energia fotoquímica e calor sensível entre a vegetação e a atmosfera são um dos principais objetivos da micrometeorologia. Um dos métodos micrometeorológicosé o Balanço de Energia - Razão de Bowen, que tem sido utilizado como padrão na determinação experimental do fluxo de calor latente (LE) e do fluxo de calor sensível, levando em consideração o saldo de radiação () e o fluxo de calor no solo (G), que são medidos diretamente por aparelhos.

Bowen (1926) introduziu o conceito de razão de Bowen como sendo a relação entre o fluxo de calor sensível $(\mathrm{H})$ e o fluxo de calor latente (LE). Esta relação pode ser expressa, de forma simplificada, como o produto do coeficiente psicrométrico pela razão entre o gradiente de temperatura do ar, o gradiente de pressão de vapor de água no ar, em pelo menos dois níveis acima da superfície.

O estudo tem como objetivo demonstrar por meio de cálculos matemáticos e princípios físicos utilizando o método de Bowen na conversão de fluxo de calor latente em unidade 
de volume de água.

\section{Desenvolvimento}

\subsection{Metodologia}

A ET foi estimada na área experimental da Reserva Particular do Patrimônio Natural do Serviço Social do Comercio - RPPN SESC - Pantanal, município de Barão de Melgaço - MT, distante $160 \mathrm{~km}$ de Cuiabá, MT, e altitude de $120 \mathrm{~m}$. Nesta área encontrava-se instalada uma torre micrometeorológica (localizada nas coordenadas 16³3'19'S, $\left.56^{\circ} 17^{\prime} 11^{\prime} \mathrm{O}\right)$ metálica de $32 \mathrm{~m}$ de altura (PEREIRA et al., 2013). O local de estudo contém aproximadamente 30 espécies, sendo que a principal espécie identificada é a Vochysia divergens Pohl (Cambará). Utilizou-se os instrumentos, saldo de radiação sobre o dossel do cambarazal foi medido por meio de um saldo radiômetro (Net Radiometer, Kipp e Zonen Delft, Inc., Holland), fluxo de calor no solo por meio de dois fluxímetros de calor no solo (HFT-3.1, REBS, Inc., Seattle, Washington). Os gradientes de temperatura e umidade do ar foram medidos por meio de dois termohigrômetros (HMP 45 C, Vaisala, Inc., Helsinki, Finland). Os dados produzidos por sinais e pulsos elétricos dos transdutores foram processados e armazenados por um datalogger (CR 10X, Campbell Scientific, Inc., Ogden, Utah), com médias de 30 minutos.

Para converter o fluxo de calor latente em unidade de volume de água foram utilizados a equação de PenmanMonteith e o método de Razão de Bowen. Para calcular o saldo de radiação foi utilizada a equação (1).

$$
\mathrm{R} \_\mathrm{n}=\mathrm{LE}+\mathrm{H}+\mathrm{G}+\mathrm{F}+\Delta \mathrm{S}+\mathrm{h}
$$

Onde é o saldo de radiação, representa o fluxo de calor latente, o calor sensível, é o calor no solo, a fotossíntese, o estoque de energia do dossel (copa das árvores) e é a advecção (transporte de energina na direção horizontal), Moore e Fisch (1986).

Sendo $\mathrm{F}<3 \%$ de $\mathrm{R} \_\mathrm{n}, \Delta \mathrm{S} \pm 3$ a $10 \%$ de $\mathrm{R} \_\mathrm{n}$ e $\mathrm{h}<2 \%$ de R_n, valores relativamente baixos, a equação (1) pode ser aproximada na equação (2).

$$
\mathrm{R}_{-} \mathrm{n}=\mathrm{LE}+\mathrm{H}+\mathrm{G}
$$

Bowen (1926) afirma que, para determinar a razão de Bowen, é necessário conhecer os valores do fluxo de calor latente e o calor sensível, conforme equações (3).

$$
\begin{gathered}
H / L E=\beta \\
H=L E . \beta
\end{gathered}
$$

Substituindo a equação (3) na equação (2), é possível determinar o fluxo de calor latente utilizando para isso o saldo de radiação, o calor do solo e a razão de Bowen, essa será usada quando o, equações (4) e (5).

$$
R_{-} n=L E+L E \cdot \beta+G
$$

$$
\begin{gathered}
\mathrm{R}_{-} \mathrm{n}-\mathrm{G}=\mathrm{LE}(1+\beta) \\
\mathrm{LE}=\left(\mathrm{R}_{-} \mathrm{n}-\mathrm{g}\right) /(1+\beta)
\end{gathered}
$$

Em termos de fluxo, o calor sensível pode ser calculado usando os dados de densidade do ar, variação de temperatura , variação de altura, calor específico do ar e o coeficiente de densidade do ar, conforme equação (6).

$$
H=\rho a \cdot C_{-} p \cdot K_{-}(H \cdot \Delta T / \Delta Z)
$$

Outra maneira de determinar o fluxo de calor latente é utilizar o coeficiente de difusividade turbulenta da água , o calor latente e a variação da umidade específica, conforme a equação (7).

$$
\mathrm{LE}=\rho \mathrm{a} \cdot \mathrm{L}_{\cdot} \cdot \mathrm{K}_{-}(\mathrm{V} .) \Delta \mathrm{q} / \Delta \mathrm{Z}
$$

Substituindo a equação de calor latente e de calor sensível na equação da razão de Bowen tem-se a equação (8).

$$
\beta=\left(\rho a C_{-}(p .) K_{-} H . \Delta T\right) /\left(\rho a . L_{.} K_{-} v \Delta q\right)
$$

Segundo a Teoria da Similaridade, (WOLF et al., 2008), pode-se determinar a razão de Bowen utilizando o calor específico do ar, a variação de temperatura, o calor latente e a umidade específica, conforme equação (9).

$$
\beta=\left(\mathrm{C}_{-} \mathrm{p} \cdot \Delta \mathrm{T}\right) /(\mathrm{L} \cdot \Delta \mathrm{q})
$$

Mas, como a umidade específica pode ser calculada com suficiente precisão pela relação $\mathrm{q}=0,622 \times \mathrm{e} / \mathrm{p}$, onde é a pressão de vapor e é a pressão atmosférica que, substituídos em (9), tem-se a equação (10).

$$
\beta=\left(\mathrm{C} \_\mathrm{p} \times \Delta \mathrm{t}\right) /(\mathrm{L} \times \Delta 0,622 \times \mathrm{e} / \mathrm{p})
$$

Assumindo como uma constante, pode- se adotar a equação (11).

$$
\beta=\left(C_{-} P \times p\right) /(L \times 0,622) \times \Delta T / \Delta \mathrm{e}
$$

Mas,

$$
(\text { C_p.p) } /(\text { L.0,622) }=\gamma
$$

onde $\gamma$ é uma constante psicrométrica que substituída na equação da razão de Bowen (11), tem-se a equação (13).

$$
\beta=(\gamma . \Delta \mathrm{T}) /\left(\Delta \mathrm{e} \_\mathrm{a}\right)
$$

A Pressão de vapor atual é fundamental para determinar a umidade relativa do ar UR, que é a relação entre a quantidade de vapor existente no ar e a que existiria se o mesmo estivesse saturado, ou seja $100 \%$ de ar saturado pelo vapor de água, na mesma temperatura, conforme equação (14).

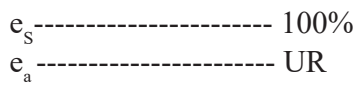




$$
\mathrm{UR}=\left(\mathrm{e} \_(\mathrm{a} .)\right. \text { 100)/e_s }
$$

Para determinar a pressão de vapor atual pode-se utilizar a equação que relaciona a umidade relativa e a pressão de vapor saturada e equação (15).

$$
\text { e_a }=(\text { UR.e_S }) / 100
$$

Através do processo de condensação que ocorre quando se torna superior a pressão de vapor saturada, que é função de temperatura. Quanto maior a temperatura, maior a sua pressão de vapor saturado, ou seja, mais água o ar pode conter. Para calcular a pressão de vapor saturado, usa-se a equação de Tetens (16).

Adotando como parâmetro $\mathrm{A}=0,61 \mathrm{kPa}$ para temperatura em ${ }^{\circ} \mathrm{C}$, tem-se as equações (16) e (17).

$$
\begin{gathered}
\text { e_S }=\mathrm{A} \times 10^{\wedge}((7,5 \mathrm{~T} / 237,3 \mathrm{~T})) \\
\mathrm{LE}=\left(\mathrm{R} \_\mathrm{n}-\mathrm{G}\right) /(1+\beta)=\mathrm{W} / \mathrm{m}^{2}
\end{gathered}
$$

Onde o (Wattes) unidade de potência por metro quadrado de área, sendo que $\mathrm{W}=\mathrm{J} / \mathrm{s}$.

\subsection{Resultados e Discussão}

\subsubsection{Cálculo da evapotranspiração}

A Evapotranspiração é a transferência de vapor d'água da superfície do solo para a atmosfera. Como o fluxo de calor latente é diretamente proporcional a massa d'água a ser evaporada e, também, diretamente proporcional a quantidade de energia destinada para que ocorra esta mudança de estado físico, ou seja calor latente de vaporização que em média tem magnitude de , 2,43MJ.kg^(-1), à $20^{\circ} \mathrm{C}$.

\section{$\mathrm{ET}=\mathrm{LE} / \mathrm{L}$}

Fazendo uma análise dimensional das unidades da no Sistema Internacional (S.I) teremos:

$$
\begin{gathered}
\mathrm{ET}=\left(\mathrm{LE}=\mathrm{W} / \mathrm{m}^{\wedge} 2\right) /(\mathrm{L} \mathrm{J} / \mathrm{kg})=\mathrm{J} /\left(\mathrm{m}^{\wedge} 2 / \mathrm{s}\right) \times \mathrm{kg} / \mathrm{J} \\
\mathrm{ET}=\mathrm{kg} /\left(\mathrm{m}^{\wedge} 2 / \mathrm{s}\right)
\end{gathered}
$$

Onde o termo se refere a massa de água, ou seja, um de água. Entretanto, a massa de um $(1,0 \mathrm{~kg})$ de água, ocupa o volume de que por sua vez representa um litro d'água. Fazendo as devidas conversões matemática tem-se.

$$
\begin{gathered}
\mathrm{ET}=\mathrm{kg} /\left(\mathrm{m}^{\wedge} 2 / \mathrm{s}\right)=1 \mathrm{dm}^{3} /\left(\mathrm{m}^{\wedge} 2 / \mathrm{s}\right)=\left(10^{\wedge}(-2) \mathrm{m}^{\wedge} 2 \times 10^{\wedge} 2 \mathrm{~mm}\right) / \\
\left(\mathrm{m}^{\wedge} 2 / \mathrm{s}\right)
\end{gathered}
$$

Esta última equação significa que evapotranspira um milimetro por metro quadrado por segundo.

$$
\mathrm{ET}=\mathrm{mm} / \mathrm{s}
$$

Para obter os valores da evapotranspiração na unidade de tempo (horária) ou diária basta fazer somatório dos valores, compreendidos no intervalo das $7 \mathrm{~h}$ às $17 \mathrm{~h}$, na unidade de tempo desejada (PEREIRA et al., 2013). Normalmente, os valores dos fluxos evapotranspirados, apresentam valores positivos no período diurno e negativos no período noturno fisicamente são fluxos ascendentes e descendentes respectivamente (PEREIRA et al., 2019)

Os valores de são descartados no período do amanhecer (5 a 7h) e no entardecer (17 a 19h), pois, nestes horários ocorrem inversão térmica entre a superfície e atmosfera. Os critérios de aceitação dos valores de estão consolidados por (PEREZ et al., 1999).

Assim, foi possível obter os dados dos fluxos de calar latente e sensível e converte-los em fluxos de massa de água ou de evapotranspiração ET Figura 1.

Figura 1 - Evapotranspiração diária (L/dia) em floresta no Cambara, entre os dias 20 de 2008 a 20 de 2009.

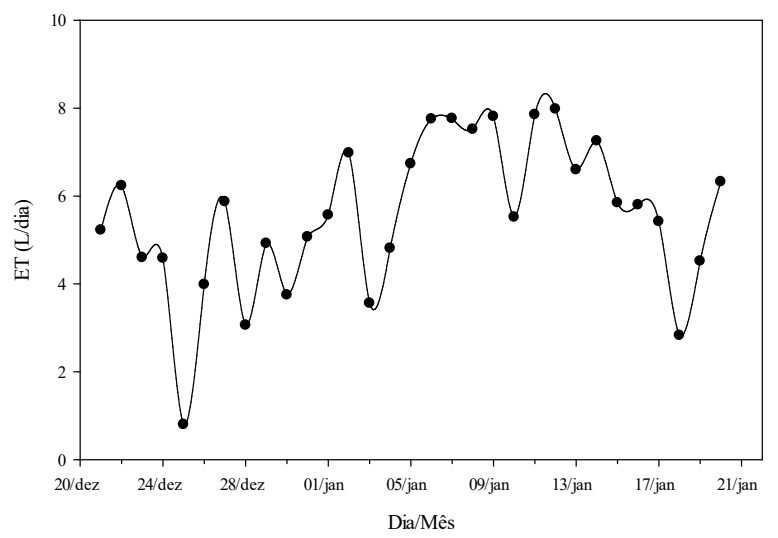

Fonte: Os autores.

Em média, a ET durante o período de estudo foi de 5,87 L/ dia ou pode ser expressa em $\left(\mathrm{mm} \mathrm{dia}^{-1}\right)$. O menor valor de ET foi no dia 25-12-2008 ( $0,80 \mathrm{~L} /$ dia $)$ e o maior valor evapotranspirado foi no dia 12-01-2009 (8,0 L/dia).

\section{Conclusão}

Durante a obtenção dos dados para se calcular a razão de Bowen, matematicamente não necessita dos valores das alturas dos sensores equações (5) e (6). Porém, fisicamente é necessária que haja gradiente de temperatura e de umidade específica do ar. O ajuste da altura dos sensores para se obter tais gradientes se dá verificando o déficit de pressão de vapor , o que normalmente se utiliza três conjuntos de sensores em níveis de altura distintos. Fazendo o entre o primeiro e o segundo, depois entre o segundo e o terceiro, a distância vertical entre os sensores será obtida havendo semelhança significativa entre os fluxos de.

\section{Referências}

ALLEN, R. G. et al. Crop evapotranspiration-Guidelines for computing crop water requirements-FAO Irrigation and drainage paper 56. Fao, v.300, n.9, p. D05109, 1998.

BATISTA DA SILVA et al. Performance of the bowen ratio-energy balance method for the estimation of reference evapotranspiration 
performance of the bowen ratio-energy balance method for the estimation of reference evapotranspiration, Irriga. v. 21, p. 516$529,2016$.

BOWEN, I. S. The ratio of heat losses by conduction and by evaporation from any water surface. Phys. Rev., v.27, n.6, p.779, 1926.

MEIRELLES, M.L. et al. Utilização do método razão de Bowen: balanço de energia na obtenção dos fluxos de vapor de agua e $\mathrm{CO}_{2}$. Embrapa Cerrados-Outras publicações técnicas (INFOTECA-E), 1999.

MONTEITH, J.L. Evaporation and surface temperature. Quarterly J. Royal Meteorol. Soc., v.107, p.1-27, 1981.

MOORE, C.J.; FISCH, G. Estimating heat storage in Amazonian tropical forest. Agricul. Forest Meteorol., v.38, p.147-169, 1986.

MU, Q.; ZHAO, M.; RUNNING, S.W. Improvements to a MODIS global terrestrial evapotranspiration algorithm. Remote Sensing Environ., v.115, n.8, p.1781-1800, 2011.
PEREIRA, O.A. et al. Determinação do fluxo de $\mathrm{CO}_{2}$ no norte do Pantanal Mato-Grossense. Rev. Bras. Meteorol., v.28, n.3, p.341352, 2013.

PEREIRA, O.A. et al. Study of $\mathrm{CO}_{2}$ Flow and Energy Balance in a Transition Forest in Southwestern Amazonia by the Modified Bowen Ratio Methods and Eddy Covariance. Australian J. Bas. Appl. Scie., 2019.

PEREZ, P.J. et al. Assessment of reliability of Bowen ratio method for partitioning fluxes. Agricul. Forest Meteorol., v.97, p.141-150, 1999.

THORNTHWAITE, C.W.; WILM, H.G.; MATHER, J.R. Report of the committee on evaporation and transpiration. Transactions Am. Geolog. Inst., v.27, n.5, p.721-723, 1946.

WOLF, A. et al. Effects of different eddy covariance correction schemes on energy balance closure and comparisons with the modified Bowen ratio system. Agricul. Forest Meteorol., v.48, p.942-952, 2008. 